\title{
POTENTIAL OF VlmybAl-2 AS A CANDIDATE MARKER FOR VISUAL IDENTIFICATION OF TRANSGENIC PLANTS: INDUCED ANTHOCYANIN PRODUCTION IN ARABIDOPSIS AND TOBACCO
}

\author{
S Geekiyanage \\ Department of Agric.Biology, Faculty of Agriculture, University of Ruhuna, Kamburupitiya
}

Accepted: $2^{\text {nd }}$ June 2009

\begin{abstract}
For development of a safe and efficient in vivo marker for plant transformation, an $m y b$-related gene of anthocyanin biosynthetic pathway, VlmybA1-2 from grape, was introduced into anthocyanin producing tobacco and Arabidopsis, and non- anthocyanin producing spinach under the control of CaMV 35S promoter. Except for the distinguishable purple color, transformed calli and plants of tobacco were not different from controls. RNA gel blot hybridization confirmed the expression of VlmybA1-2 in purple tobacco seedlings. Completely purple T1 Arabidopsis seedlings could not survive as high anthocyanin levels may affect normal growth; Surviving T1 seedlings could produce viable seeds of two distinguishable colors: purple and brown (similar to wild type). Purple seeds germinated on kanamycin medium providing an easy method of transgenic seed identification in Arabidopsis. T2 and T3 completely purple seedlings produced purple flowers and seeds. Putative transgenic spinach was not different to control in color, although presence of VlmybA1-2 was confirmed by DNA gel blot hybridization. VlmybA alone, without the aid of an myc-related gene partner, could induce complete pigmentation in tobacco and Arabidopsis indicating its potential over previously used $m y b$ - and $m y c$-related genes.
\end{abstract}

Key words: Anthocyanin, Arabidopsis, In vivo marker, Tobacco, VlmybAl-2

\section{INTRODUCTION}

Anthocyanins are unique pigments in flowering plants. Structural genes encoding the enzymes of the anthocyanin biosynthetic pathway are conserved among different species (Holton and Cornish 1995). Two classes of regulatory genes, $m y b$ and $m y c$ of $C$ and $R$ families activate the transcription of structural genes in maize. Differences in the regulatory mechanisms controlling the expression of structural genes, and interactions among them would account for the intensity and variety of pigmentation patterns among different plant species. Therefore, the manipulation of regulatory genes in transgenic plants may be versatile over that of structural genes.

Several regulatory genes from $C$ and $R$ families have been isolated from several plants and one of such gene alone could not induce distinguishable phenotypes in transgenic plants (Lloyd et al. 1992). Three types of $m y b$-related genes capable of anthocyanin induction in non-colored grape have been isolated from Kyoho grape: VlmybA 1-1, VlmybA1-2 and VlmybA2 (Kobayashi et al. 2002). VlmybA genes have a unique protein sequence compared with other known plant $m y b$-related genes of the anthocyanin biosynthetic pathway (Kobayashi et al. 2002). VlmybA2 could induce anthocyanin pigmentation in Arabidopsis and tobacco in a previous attempt (Geekiyanage et al. 2007).
Development of alternatives for commonly used marker genes, conferring resistance to antibiotics and herbicides, is useful for safer genetically modified crops to maximize their benefits. If such genes would not affect the normal plant growth and development of the host plant, enhanced anthocyanin production induced by them could be exploited for a simple, non-destructive, efficient identification of transformation at early stages and to investigate the potential of out-crossing of GM crops in the field.

Enhanced anthocyanin production, induced by regulatory genes from maize have been reported in maize, Arabidopsis, tobacco and tomato, and their potential as visual markers has been highlighted in those cases (Ludwig et al. 1990; Lloyd et al. 1992; Goldsbrough et al. 1996; Tamaoki et al. 2006).

Anthocyanin pigments are useful in human health in a variety of fields and in food industry as a natural food colorant as well (Lila 2004). Attempts to develop callus and plant cell systems from a variety of crops have been made to produce stable anthocyanin pigments in vitro to be used for food industry and bio-medicinal purposes. Genetically engineered callus or cell lines must produce high pigment yield and their dependence on irradiance can be low.

In this study, I examined the potential of a grape $m y b$-related gene, VlmybAl-2, to induce anthocyanin biosynthesis in anthocyanin producing plants, tobacco and Arabidopsis, which are previ- 
ously tested for some $m y b$-related genes and in nonanthocyanin producing spinach, in order to check the functional equivalence of an anthocyanin regulatory gene of a dicot, grape, in them. This was an attempt for the future work of development of a safe in vivo marker for plant transformation studies and development of plant cell systems that produce stable anthocyanins for applications as natural food colorants in bio-industry.

\section{METHODOLOGY}

\section{Plant materials, growth conditions and culture media}

Arabidopsis thaliana ecotype Columbia, Nicotiana tabacum cultivar Maryland Mammoth and Spinacia oleracia L. cultivar Longstanding Bloomsdale Dark Green were used for the experiment. Growth conditions and culture media for Arabidopsis and tobacco were as previously described (Geekiyanage et al. 2007). Spinach seedlings were grown on MS medium (Murashige and Skoog 1962) under the short-day (SD) condition (8h light and 16h darkness) for cotyledon explants.

\section{Agrobacterium-mediated transformation}

Agrobacterium tumefaciens strain LBA4404 harboring the pBI121 vector carrying full-length cDNA of VlmybA1-2 coding region, cloned by XbaI and SacI sites at 5' and 3' ends respectively (Kobayashi et al. 2002) in place of the GUS gene under the control of the CaMV $35 \mathrm{~S}$ promoter, and NPTII gene under NOS promoter were used for the transformation.

Arabidopsis inflorescences were transformed by the floral dip method (Clough and Bent 1998). The method of Horsch et al. (1985) was used for tobacco leaf disc transformation. T1 seeds were selected on kanamycin-containing medium.

Spinach cotyledons were transformed according to the method of Zhang and Zeevaart (1999).

\section{RNA gel blot hybridization}

RNA gel blot hybridization was performed as previously described (Geekiyanage et al. 2007).

Identification and measurement of anthocyanin Identification of anthocyanin was performed as previously described (Geekiyanage et al. 2007).

\section{RESULTS}

Different phenotypes of 35S: VlmybA1-2 T1 Arabidopsis seedlings

There were five $\mathrm{T} 1$ seedlings that showed three phenotypes (Fig 1): two seedlings had purple roots

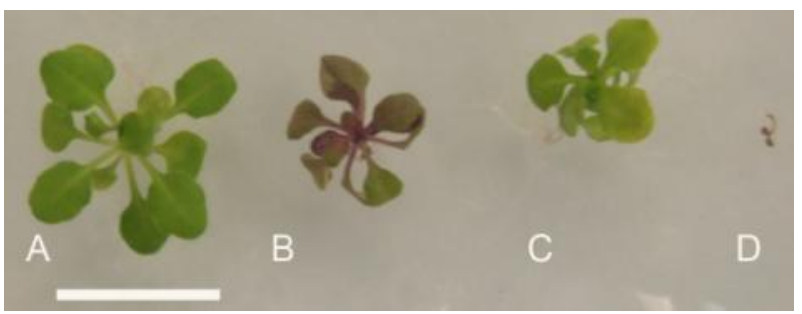

Figure 1 Different phenotypes of T1 Arabidopsis plants of 35S:VlmybA1-2 after 2 weeks of germination. (A) A seedling with purple-green leaves and purple roots (B) A completely purple seedling (C) A seedling with green leaves and purple roots (D) A completely purple seedling which could not grow beyond cotyledon stage. $\mathrm{Bar}=2 \mathrm{~cm}$

and, purple-green leaves showing normal growth and flowered and set seeds of two distinguishable colors; purple and brown similar to wild type; three seedlings were completely purple, out of two could not grow beyond the cotyledon stage, while the other was transferred to a pot and died before flowering.

\section{Segregation of VlmybA1-2 in T2 seeds of Arabidopsis}

Seeds of two independent T1 lines showed two phenotypes: purple and brown seeds, similar to wild type (Fig 2). The ratio of purple to brown seeds was 3:1 suggesting that homozygous and heterozygous seeds for the transgene are purple in color (data not shown). It was confirmed by the germination test for purple and brown seeds on kanamycin containing medium and kanamaycinfree medium (Fig 3).

The purple pigment from Arabidopsis could be extracted with water and became dark blue with the addition of $0.1 \mathrm{M}$ sodium hydroxide. These results suggest that the pigment is anthocyanin (Rabino and Mancinelli 1986; Uimari and Strommer 1998; Kobayashi et al. 2005).

VlmybA1-2 induced anthocyanin production in T2 and T3 Arabidopsis plants

Purple T2 seeds of completely purple T1 plant germinated on kanamycin-containing medium. Germi-

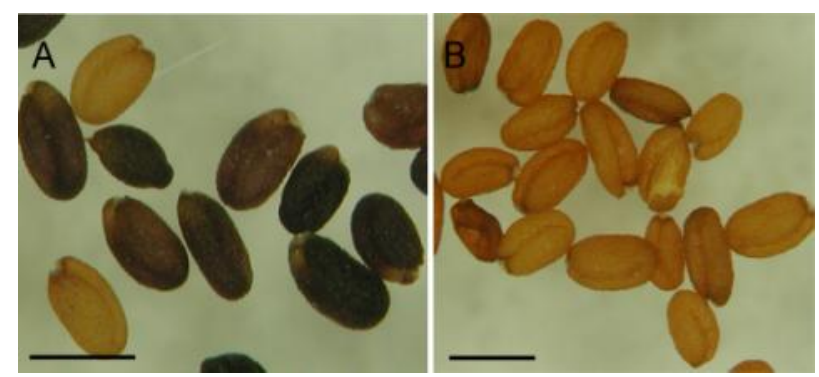

Figure 2 Segregating seed coat color of Arabidopsis in T2 generation. (A) Seeds of 35S:VlmybA1-2 plant showing two colors, purple and brown. Brown seeds were similar to wild type seeds in color (B) Wild type. Bar= $1 \mathrm{~mm}$ 


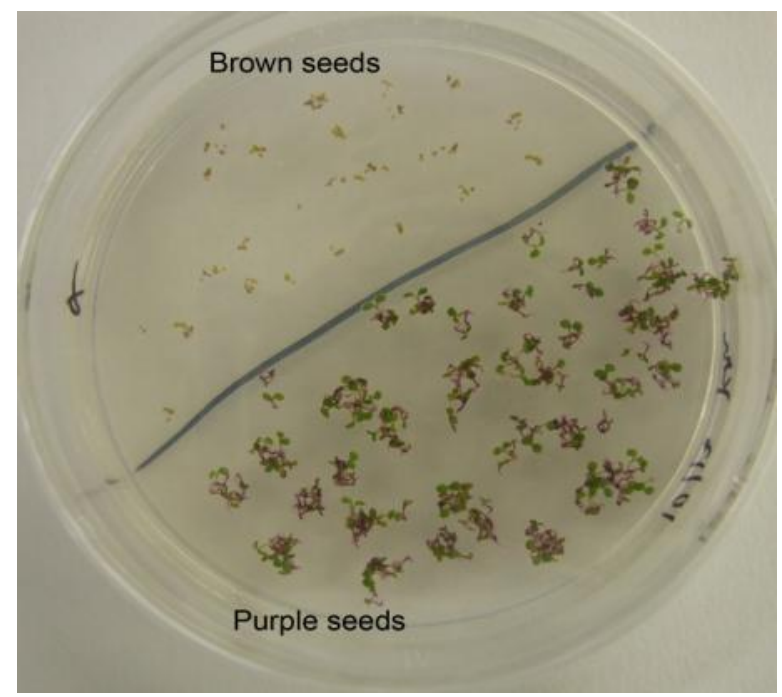

Figure 3 Co-segregation of VlmybA1-2 and kanamycin resistance in T2 Arabidopsis seeds on MS medium after one week of seed germination. (A) Purple seeds could germinate on $50 \mathrm{mg} \mathrm{1-1}$ kanamycin containing MS medium (B) Brown seeds could not germinate on kanamycin containing MS medium.

nating seedlings were with green leaves and purple roots. Some of them produced all purple T3 seeds while some produced both purple and brown seeds. Germinating all purple seeds and some purple seeds of both colors, produced completely purple plants, out of which could produce purple flowers (Fig 4; A and D), plants with purple-green leaves and well grown purple roots, which could produce white flowers (Fig 4; B and E) and plants with translucent green leaves and purple roots (Fig $4 ; \mathrm{C}$ ).

\section{Plant regeneration from transgenic tobacco callus}

Purple protrusions originated from leaf explants after 2 weeks of transformation by the pBI121 vector carrying VlmybA1-2 cDNA. Purple callus proliferated from such purple protrusions (Fig 5) and purple shoots regenerated from them. Purple roots were subsequently regenerated and were distinguishable from plants regenerated from wild type or control vector transformants by color (Fig 6). The transgenic plants grew normally and produced flowers (Fig 7). The corolla of the transgenic flow-
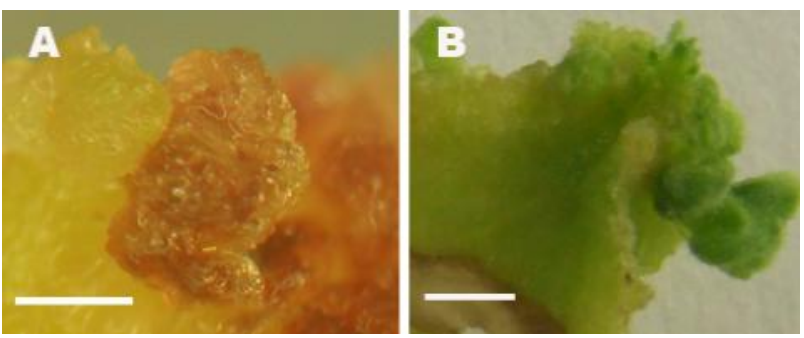

Figure 5 Expression of VlmybA1-2 in transformed tobacco leaf derived callus. (A) 35S:VlmybA1-2. (B) Wild type. Bar = $1 \mathrm{~mm}$.

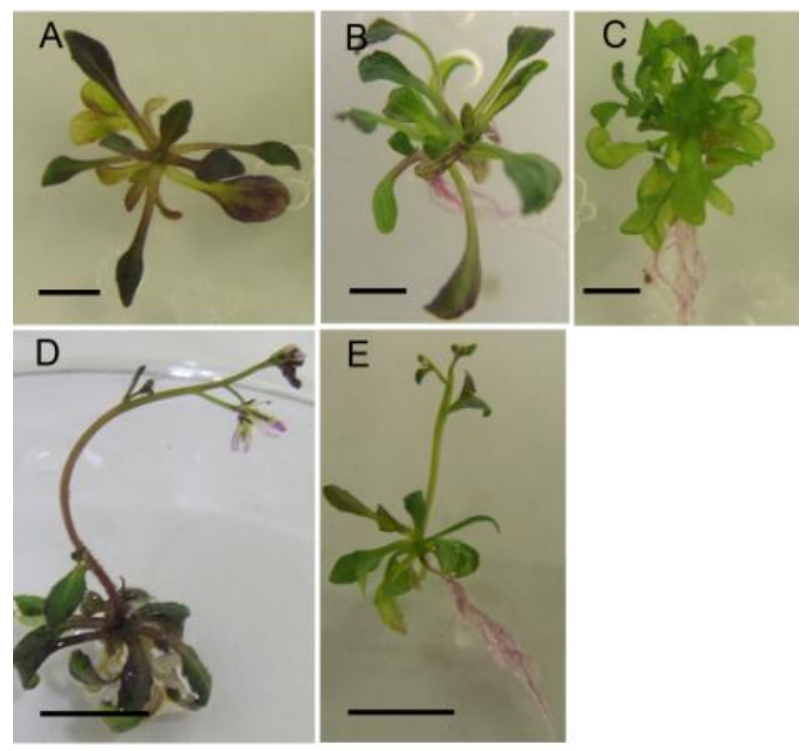

Figure 4 Different phenotypes of T3 Arabidopsis plants of 35S:VlmybA1-2 after 3 weeks of seed germination. (A) A completely purple plant (B) A plant with purple-green leaves and purple roots (C) A plant with abnormal green leaves and purple roots (D) A purple plant with purple flowers at flowering $(\mathrm{E}) \mathrm{A}$ purple-green plant at flowering. $\mathrm{Bar}=$ $1 \mathrm{~cm}$

ers was dark purple and distinguishable from the light pinkish corolla of wild type flowers.

\section{VlmyA1-2 expression in transgenic tobacco plants}

Five independent transgenic regenerants were checked for transgene expression by RNA gel blot hybridization. All transgenic regenerants expressed high levels of the transgene (Fig 8).

\section{Segregation of VlmybA1-2 in T1 seedlings of to- bacco}

Primary transgenic plants produced fertile $\mathrm{T} 1$ seeds. Kanamycin resistance and VlmybA1-2induced purple color were co-expressed. Surviving seedlings were completely purple and greenish purple. Completely green seedlings could not grow beyond the cotyledon stage and died on kanamycin-
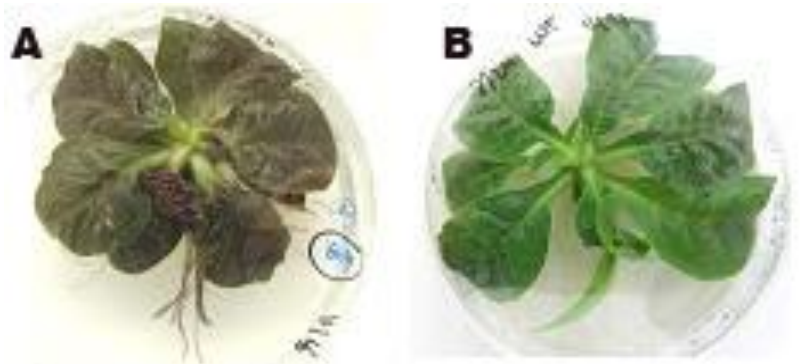

Figure 6 A 35S:VlmybA1-2 tobacco plant regenerated from leaf callus on root formation medium. (A) A 35S:VlmybA1-2 transgenic plant with purple leaves and purple roots was distinguishable from a control vector transformant (B). 


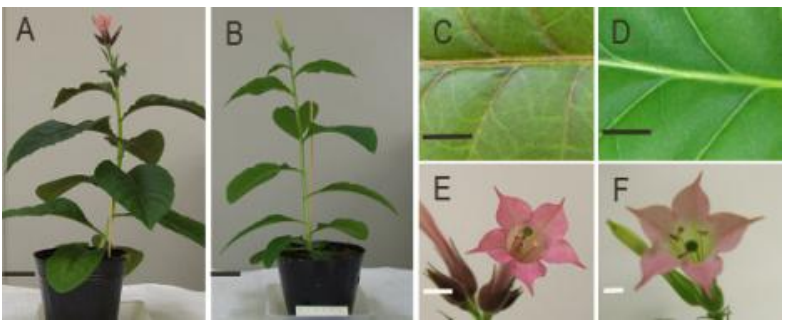

Figure 7 A regenerated 35S:VlmybA1-2 tobacco plant grown under the SD condition in a pot (A). Flowering occurred around 2 months after pot transfer. Bar $=4 \mathrm{~cm}$. (C) A magnified view of a part of a $35 \mathrm{~S}: \mathrm{VlmybA1}-2$ leaf. $\mathrm{Bar}=5$ mm. (E) A 35S:VlmybA1-2 flower. Bar $=1 \mathrm{~cm}$. (B)(D) and (F) Wild type.

containing medium (Fig 9). Ratio between surviving completely purple and greenish purple seedlings to dead green seedlings was 53:17 (approximately 3:1) in an observed progeny of a line (line one), indicating that the Mendelian inheritance of a single gene locus.

\section{$V l m y b A$ induced anthocyanin production in tobacco}

The purple pigments from tobacco leaf callus, regenerated plants and T1 seedlings could be extracted with water and became dark blue with the addition of $0.1 \mathrm{M}$ sodium hydroxide. These results suggest that the pigment is anthocyanin.

The absorbance values at $525 \mathrm{~nm}$ of the acetic acid extracts per $\mathrm{g}$ fresh weight tissue were interpreted as the anthocyanin content. The absorbance values were 235.51 \pm 12.31 (standard error, $n=3$ ) and $250.31 . \pm 20.45(\mathrm{SE}, \mathrm{n}=3)$ in purple line 1 plants and line 2 plants, respectively, while they were $153.14 \pm 1.47(\mathrm{SE}, \mathrm{n}=3)$ and $136.67 \pm 7.18(\mathrm{SE}, \mathrm{n}=3)$ in greenish purple line 1 plants and line 2 plants, respectively. An average absorbance of $1.42 \pm 0.19$

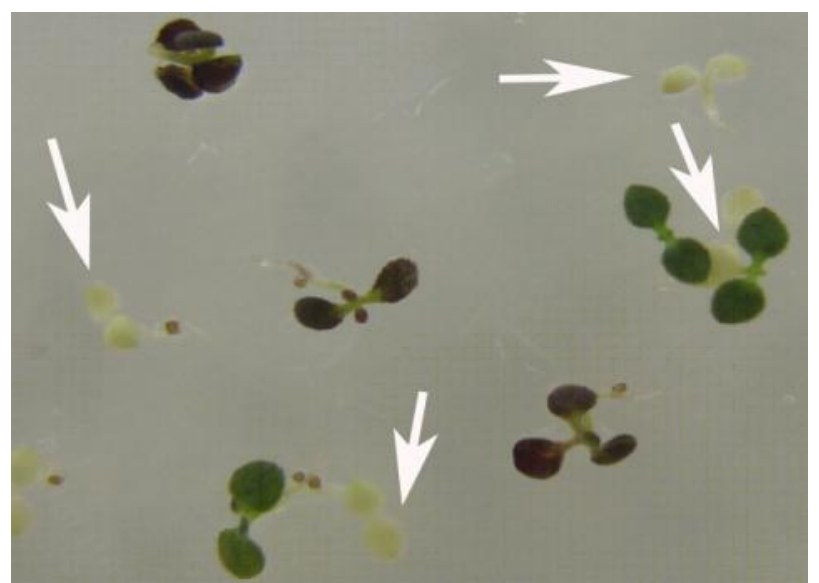

Figure 9 Segregation of 35S:VlmybA1-2 tobacco seedlings of $\mathrm{T} 1$ generation on kanamycin-containing medium. Complete purple seedlings and seedlings with partially purplegreen leaves could survive on kanamycin containing medium. White arrows indicate dead seedlings. These plants are derived from the primary transformant corresponding to line 2 in Figure 4.

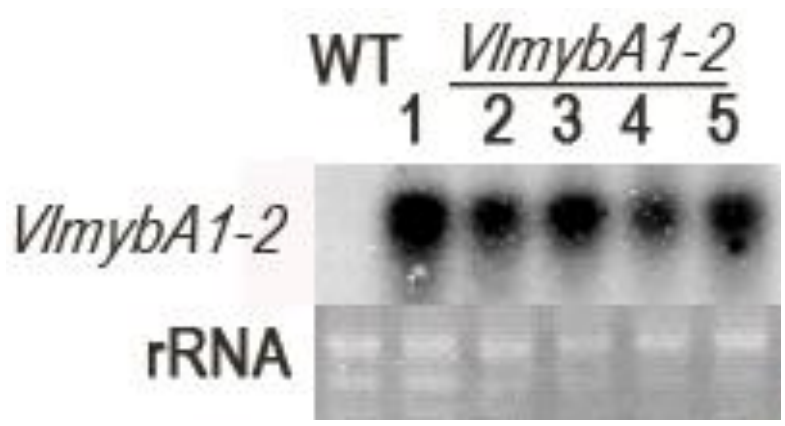

Figure 8 RNA gel blot hybridization of tobacco primary transformants. WT: Wild type. Numbers 1 to 5 are blots for five regenerated independent 35S:VlmybA1-2 plant lines.

$(\mathrm{SE}, \mathrm{n}=3)$ at $525 \mathrm{~nm}$ was recorded from the wild type.

\section{VlmybA1-2 could not induce anthocyanin production in spinach}

Both infected and wild type spinach cotyledon bases were purple after 3 days of culture, giving no indication for transformed tissue identification. A few regenerated putative transgenic spinach plants, similar to wild type in color and appearance, could survive on kanamycin- containing medium (data not shown) and the presence of VlmybAl-2 was confirmed by DNA gel blot hybridization in one of them, which could survive on $50 \mathrm{mg} \mathrm{l}^{-1}$ kanamycincontaining medium for more than 4 months (Fig 10): number of regenerated plants after transformation was limited due to low regeneration frequency of spinach.

\section{DISCUSSION}

The over-expression of VlmybA1-2 alone could induce anthocyanin pigmentation in whole plant in Arabidopsis and tobacco transformants. As VlmybA are transcription factor genes in the anthocyanin biosynthetic pathway in a dicot, it may bind to the promoters of the structural genes of the anthocyanin biosynthetic pathway in them, which may be conserved among anthocyanin-producing dicots.

\section{Regenerated plants + -}

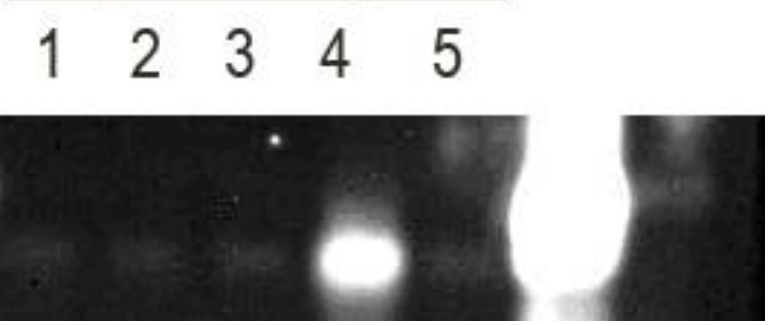

Figure 10 DNA gel blot hybridization of spinach primary transformants: Numbers 1 to 5 are blots for five regenerated independent putative 35S:VlmybA1-2 plants. (+) Positive control. (-) Wild type. 
Together with the previous result of VlmybA2 (Geekiyanage et al.2007), and results of present study for Arabidopsis and tobacco, it is postulated that VlmybA genes can function in a variety of anthocyanin-producing dicots.

The copy number of the transgene or the localization of transgene insertion in the genome may determine the level of transgene expression resulting in phenotypic differences in T1. Furthermore, all T3 Arabidopsis purple seeds and some of T2 purple seeds derived from both color T1 plants, produced completely purple plants including flowers. Normal plant growth was disturbed in some T1 transformants of Arabidopsis: Two completely purple seedlings did not survive beyond the cotyledon stage, while the surviving partially purple seedlings with purple roots produced seeds that segregated according to the expected Mendelian ratio of 3:1 for the inheritance of a single gene locus. Furthermore, the completely purple T1 seedlings showed retarded root growth or had no roots at all. A similar phenotype was lethal in tomato transformed by Lc (Goldsbrough et al. 1996). Flavonoids act as negative regulators of auxin transport in Arabidopsis (Brown et al. 2001). The effect on root formation could be due to the negative regulation of auxin transport by the over-expressed flavonoids in Arabidopsis.

$V l m y b A$ genes induce remarkably the expression of the UFGT gene, while the expression of the other anthocyanin structural genes is slightly increased in grape (Kobayashi et al. 2002). In Arabidopsis, the color of testa is mainly due to the oxidation of proanthocyanidins (Chapple et al. 1994). In the present study, the pigmentation in Arabidopsis seeds, making it a distinguishable phenotype, could be due to the enhanced production of anthocyanin or proanthocyanidins. This distinguishable phenotype could be observed in 35S: VlmybA2 as well (Geekiyanage et al. 2007) and suggests that in Arabidopsis, the VlmybA2 and VlmybA1-2 gene product/s may activate not only UFGT but also other structural genes that are upstream or at the branched end towards proanthocyanidin in seed coat. Due to this phenotype, VlmybA genes provided an efficient and simple method for the identification of transformed seeds in Arabidopsis.

VlmybA1-2 expression in Arabidopsis seems to be promising for visual identification of transformants as all T1 transformants and their progenies showed distinguishable purple color to the wild type. Plants with green leaves and normal growth were even distinguishable by purple roots, and ability to induce purple color in roots is a unique feature in VlmybA genes compared to previously used other $m y b$ or $m y c$ genes. Furthermore, although anthocyanin pigmentation can be induced by increased irradiation or stress in other parts of the plant (Rabino and Mancinelli 1986), there are no reports on such pigmentation in roots. Expression of VlmybAl-2 in seeds provided an efficient and unique method of transgenic seed identification in Arabidopsis. However, level of expression may affect the normal growth in Arabidopsis.

Numerous environmental, developmental and tissue- and cell-specific factors are involved in anthocyanin biosynthesis In the present work, the distribution and intensity of purple color of the entire regenerated tobacco shoots were affected as purple color was prominent in the veins of leaf upper surface and lower leaf blade of pot-grown plants (Fig 7). The differences in anthocyanin distribution in the leaves of 35S:VlmybA1-2 plants could have resulted from the availability of endogenous factors, which may be dependant on developmental stage,and/or on environmental conditions.

Anthocyanin content in 35S:VlmybAl-2 tobacco calli/ plants were several times higher than the control, indicating its potential for development of plant cell systems that produce stable anthocyanins for applications as natural food colorants in bio-industry.

Spinach belongs to Caryophyllales, which produce betacyanins instead of anthocyanins. Anthocyanin and betacyanins are produced in two mutually exclusive pathways in plants (Stafford, 1994). The presence of the first committed structural gene in anthocyanin biosynthetic pathway, dihydroflavonol 4-reductase $(D F R)$, in betalain-producing plants suggests that the lack of transcription factor genes could be a reason for inability to produce anthocyanin in them (Shimada et al 2004). VlmybA genes (Kobayashi et al. 2002) and previously cloned regulatory genes such as $\mathrm{Cl}$ (Cone et al. 1986; Paz-Ares et al. 1987), AN2 (Quattrocchio et al. 1998), DELILA (Goodrich et al. 1992), Lc (Ludwig et al. 1990), AN11 (Quattrocchio et al. 1993) and TTG1 (Shirley et al. 1995) would provide a means to test this hypothesis in transgenic betalain-producing plants. Lack of distinguishable pigmentation from control in the VlmybAl-2 spinach plant and regenerated shoots on kanamycin medium, may be due to the inability of VlmybAl-2 to induce structural genes of anthocyanin biosynthesis pathway in non anthocyanin producing spinach (Shimada et al. 2007).

VlmybA1-2 expression in tobacco seems to be promising for visual identification of transformants as all tobacco primary transformants and their progenies are apparently not different from the wild type except for the purple color. Flavonoids act as negative regulators of auxin transport in Arabidopsis (Brown et al. 2001). Negative effect on normal plant growth by over-expressed flavonoids in Arabidopsis was not observed in transgenic tobacco plants. 
Most commonly used markers are the bacterial genes conferring resistance to antibiotics kanamycin and hygromycin or to herbicide glufosinate ammonium. There is a growing social concern over use of these genes in transgenic plants in eco systems and human consumption. In addition, there are some evident drawbacks of use of these genes because selection chemicals, like antibiotics may either interfere with plant regeneration, resulting in a fewer transgenic plants or may be less effective as plant tissues may exhibit a high level of intrinsic antibiotic resistance, which is misleading during selection.

A versatile gene for anthocyanin production could be a good candidate for a simple and nondestructive visual marker for plant transformation and may replace controversial antibiotic marker genes. VlmybA genes show higher potential than the other anthocyanin regulatory genes previously tested (Ludwig et al. 1990; Lloyd et al. 1992; Mooney et al. 1995; Goldsbrough et al. 1996; Deluc et al. 2006). Its potential should be exploited in the genetically modified plant production process, starting from the efficient recovery of regenerants during transformation up to the monitoring of transgenic plants at field level for risk assessment, and development of plant cell systems that produce stable anthocyanins for applications as natural food colorants in bio-industry.

\section{CONCLUSIONS}

- 35S:VlmybA1-2 can induce anthocyanin pigmentation in whole plants of tobacco and Arabidopsis.

- High levels of anthocyanin production are associated with lethality and abnormality in Arabidopsis seedlings.

- Color of 35S:VlmybA1-2 Arabidopsis seeds can be used for transgenic seed identification.

- VlmybA1-2 shows a potential to be used as a visual marker for anthocyanin producing plants

\section{Acknowledgements}

I gratefully acknowledge Prof. Tomohiro Kiyosue for supervision and kind permission for publishing the work, and Japanese government for financial support. I thank Dr S Kobayashi, Department of Grape and Persimmon Research, National Institute of Fruit Tree Science, Hiroshima, Japan, for the kind gift of pBI121 vector containing VlmybA1-2, Ms K Asayama for technical assistance, and Dr M Kyo, Faculty of Agriculture, Kagawa University, for tobacco plant material .

This work was partly supported by a grant to Prof. Tomohiro Kiyosue from the Program for Pro- motion of Basic Research Activities for Innovative Biosciences (PROBRAIN).

\section{REFERENCES}

Brown DE, Rashotte AM, Murphy AS, Normanly J, Tague BW, Peer WA, Taiz L and Muday GK 2001 Flavonoids act as negative regulators of auxin transport in vivo in Arabidopsis. Plant Physiol. 126: 524-535.

Chapple CCS, Shirley BW, Zook M, Hammerschmidt R and Somerville SC 1994 Secondary metabolism in Arabidopsis. In: Arabidopsis, Cold Spring Harbour Laboratory Press, New York, pp. 989-1030.

Clough SJ and Bent AF 1998 Floral dip: a simplified method for Agrobacterium-mediated transformation of Arabidopsis thaliana. Plant J. 16: 735-743.

Cone KC ,Burr F and Burr B 1986 Molecular analysis of the maize anthocyanin regulatory locus C1. Proc Natl Acad Sci USA. 83: 96319635.

Consonni G, Geuna F, Gavazzi G and Tonelli C 1993 Molecular homology among members of the $\mathrm{R}$ gene family in maize. Plant J. 3: 335-346.

Deluc L, Barrieu F, Marchive C, Lauvergeat V, Decendit A, Richard T, Carde J, Merillon J and Hamdi S 2006 Characterization of grapevine R2R3-MYB transcription factor that regulates the phenylpropanoid pathway. Plant Physiol. 140: 499-511.

Geekiyanage S, Takase T, Ogura Y and Kiyosue T 2007 Anthocyanin production by grape transcription factor gene VlmybA2 in transgenic tobacco and Arabidopsis. Plant Biotech Rep. DOI 10.1007/s 11816-006-0001-4 (online publication)

Goldsbrough AP, Tong Y and Yoder JI 1996 Lc as a non-destructive visual reporter and transposition excision marker gene for tomato. Plant J. 9:927-933.

Goodrich J, Carpenter R and Coen ES 1992 A common gene regulates pigmentation pattern in diverse plant species. Cell. 68: 955-964.

Holton TA and Cornish EC 1995 Genetics and biochemistry of anthocyanin biosynthesis. Plant Cell. 7: 1071-1083.

Horsch RB, Fry JE, Hoffmann NL, Eichholtz D, Rogers SG and Fraley RT 1985 A simple and general method for transferring genes into plants. Science. 227: 1229-1231.

Kobayashi S, Goto-Yamamoto N and Hirochika $\mathrm{H}$ 2005 Association of $V v m y b A l$ gene with anthocyanin production in grape (Vitis vinifera) skincolor mutants. J Jpn Soc Hortic Sci. 74: 196203. 
Kobayashi S, Ishimaru M, Hiraoka K and Honda C $2002 M y b$-related genes of the Kyoho grape (Vitis labruscana) regulate anthocyanin biosynthesis. Planta. 215: 924-933.

Lila MA 2004 Anthocyanins and human health: An in vitro investigative approach.J Biomed Biotechnol. 5:306-313.

Lloyd AM, Walbot V and Davis RW 1992 Arabidopsis and Nicotiana anthocyanin production activated by maize regulators $\mathrm{R}$ and $\mathrm{C} 1$. Science. 258: 1773-1775.

Ludwig SR, Bowen B, Beach L and Wessler SR 1990 A regulatory gene as a novel visible marker for maize transformation. Science. 247: 449-450.

Mooney M, Desnos T, Harrison K, Jones J, Carpenter R and Coen E 1995 Altered regulation of tomato and tobacco pigmentation genes caused by the DELILA gene of Antirrhinum. Plant J. 7: 333-339.

Murashige T and Skoog F 1962 A revised medium for rapid growth and bioassays with tobacco tissue cultures. Physiol Plant. 15: 473-497.

Paz-Ares J, Ghosal D, Wienand U, Peterson PA and Saedler H 1987 The regulatory $c 1$ locus of Zea mays encodes a protein with homology to $m y b$ proto-oncogene products and with structural similarities to transcription activators. EMBO J. 6: 3553-3558.

Quattrocchio F, Wing JF, Leppen HTC, Mol JNM and Koes RE 1993 Regulatory genes controlling anthocyanin pigmentation are functionally conserved among plant species and have distinct sets of target genes. Plant Cell. 5: 14971512.

Quattrocchio F, Wing JF, van der Woude K, Mol JNM and Koes R 1998 Analysis of bHLH and MYB domain proteins: species-specific regulatory differences are caused by divergent evolu- tion of target anthocyanin genes. Plant J. 13: 475-488.

Rabino I and Mancinelli AL 1986 Light, temperature and anthocyanin production. Plant Physiol 81: 922-924.

Shimada S, Takahashi K, Sato Y and Sakuta M 2004 Dihydroflavonol 4-reductase cDNA from non-anthocyanin producing species in Caryophyllases. Plant Cell Physiol. 45: 1290-1298.

Shimada S, Otsuki H and Sakuta M 2007 Transcriptional control of anthocyanin biosynthetic genes in Caryophyllales. J Experimental Botany. 58: 957-967.

Shirley BW, Kubasek WL, Storz G, Bruggemann E, Koornneef M, Ausubel FM and Goodman HM 1995 Analysis of Arabidopsis mutants deficient in flavonoid biosynthesis. Plant J. 8: 659 -671 .

Stafford HA 1994 Anthocyanins and betalains: evolution of the mutually exclusive pathways. Plant Sci. 101: 91-98.

Tamaoki M, Imai H, Takahashi H, Toda Y, Niwa Y, Nakajima N, Aono M, Kubo A and Saji H 2006 Development of visible markers for transgenic plants and their availability for environmental risk assessment. Z. Naturforsch. 377386.

Uimari A and Strommer J 1998 Anthocyanin regulatory mutations in pea: effects on gene expression and complementation by $R$-like genes of maize. Mol Gen Genet. 257: 198-204.

Zhang HX and Zeevaart JAD 1999 An efficient Agrobacterium tumifaciens-mediated transformation and regeneration system for cotyledons of spinach (Spinacia oleracea L.). Plant Cell Rep.18: 640-645. 